Supporting Information for

\title{
Naphthalenediimide Cations Inhibit 2D Perovskite Formation and Facilitate Subpicosecond Electron Transfer
}

Andrew H. Proppe, ${ }^{1,2}$ Marie-Hélène Tremblay, ${ }^{3}$ Yadong Zhang, ${ }^{3}$ Zhenyu Yang, ${ }^{2, \S}$ Rafael

Quintero-Bermudez, ${ }^{2}$ Shana O. Kelley, ${ }^{1,4}$ Stephen Barlow, ${ }^{3}$ Seth R. Marder, ${ }^{3}$ Edward H. Sargent $* 2$

1 Department of Chemistry, University of Toronto, Toronto, Ontario, Canada, M5S 3G4

2 The Edward S. Rogers Department of Electrical and Computer Engineering, University of Toronto, Toronto, Ontario, Canada, M5S 3G4

3 Center for Organic Photonics and Electronics, School of Chemistry and Biochemistry, Georgia Institute of Technology, Atlanta, USA

4 Department of Pharmaceutical Sciences, Leslie Dan Faculty of Pharmacy, University of Toronto, Toronto, Ontario, Canada, M5S 3M2

\section{Contains}

- Materials

- Supplementary Figures: S1 - S12 


\section{Materials}

Materials: $\mathrm{PbI}_{2}$ (Alfa Aesar, 99.999\%, ultra dry), methylammonium iodide (MAI, Dyesol), phenethylammonium iodide (PEAI, Dyesol), dimethylsulfoxide anhydrous (DMSO, 99.\%, Sigma), $\gamma$-Butyrolactone (GBL, >99\%, Sigma), chlorobenzene anhydrous (CB, Sigma), N,Ndimethylformamide (DMF; anhydrous, 99.8\%, Sigma). 2,2'-Naphthalene-1,8:4,5bis(dicarboximide)- $N, N^{\prime}$-diyl]-bis(ethyammonium) diiodide (NDIC2- $\mathrm{I}_{2}$ ) was synthesized according to our recent report. ${ }^{1} \quad 4,4^{\prime}$-Naphthalene-1,8:4,5-bis(dicarboximide)- $N, N^{\prime}$-diyl]bis(butylammonium) diiodide (NDIC4- $\mathrm{I}_{2}$ ) was synthesized in an analogous fashion, but we found that this salts was poorly soluble in DMF and DMSO, preventing us from coating sufficiently thick films for optical and structural characterization.

Table S1. Mass of precursors dissolved in $1 \mathrm{~mL}$ of DMF to obtain $\left[\mathrm{Pb}^{+2}\right]=0.8 \mathrm{M}$ solutions

\begin{tabular}{c|c|c|c|c}
$\langle\boldsymbol{n}\rangle$ & PbI $\mathbf{2}(\mathbf{m g})$ & MAI (mg) & FAI (mg) & NDIC2 (mg) \\
\hline $5(\mathrm{MA}: F A)$ & 368.80 & 93.57 & 15.26 & 97.31 \\
\hline $5(\mathrm{MA})$ & 368.80 & 0.00 & 101.76 & 97.31 \\
\hline 10 & 368.80 & 0 & 114.48 & 48.65 \\
\hline 20 & 368.80 & 0 & 120.84 & 24.33 \\
\hline $\mathrm{MAPbI}_{3}$ & 368.80 & 0 & 0 & 0
\end{tabular}




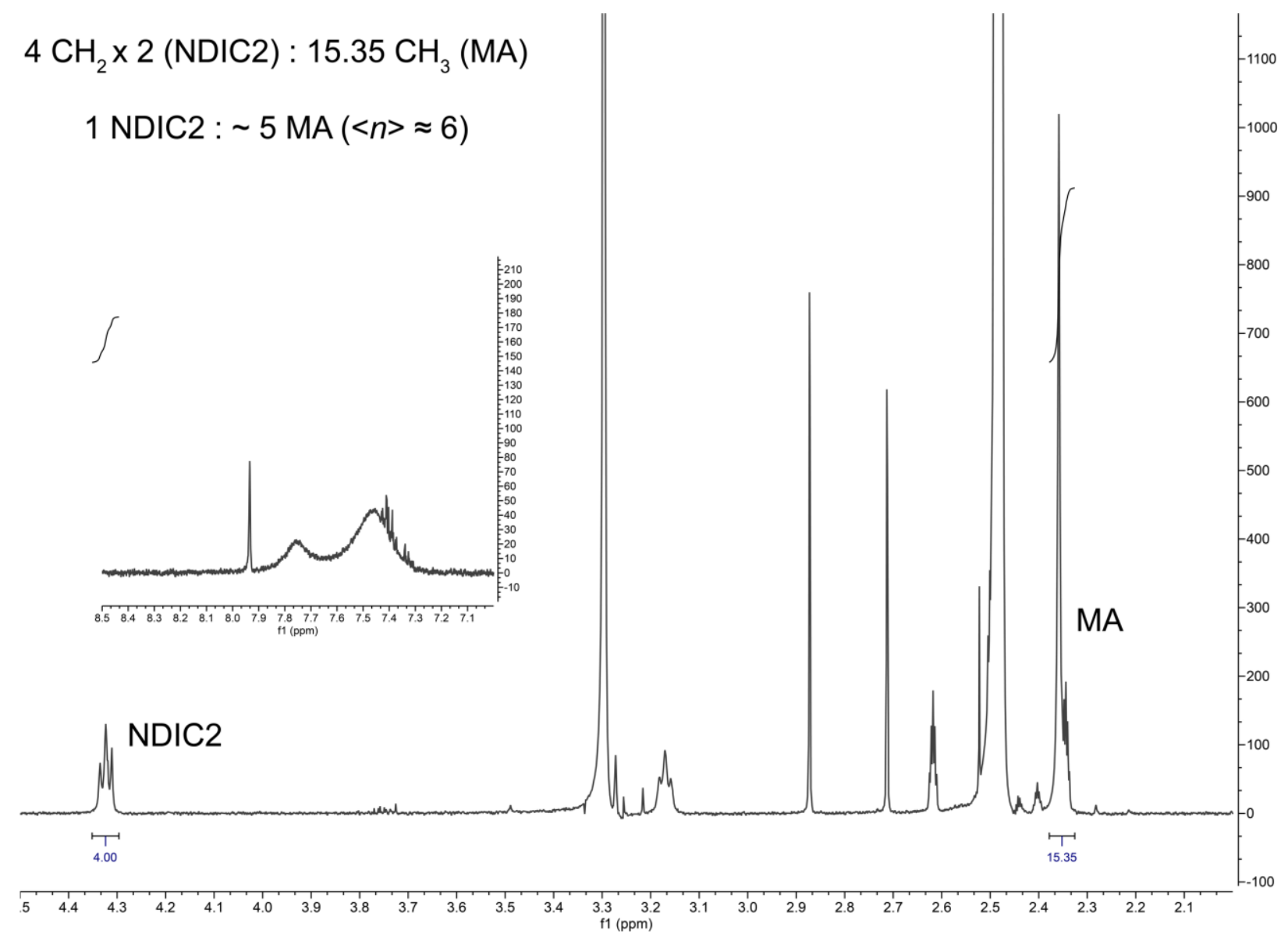

Figure S1. ${ }^{1} \mathrm{H}-\mathrm{NMR}$ spectrum of an $\langle n\rangle=5$ film with nominal formula (NDIC2)(MA $\left.{ }_{4}\right) \mathrm{Pb}_{5} \mathrm{I}_{16}$ dissolved in $d^{6}$-DMSO, and calculation of in-film $\langle n\rangle$ based on the cation ratio. 


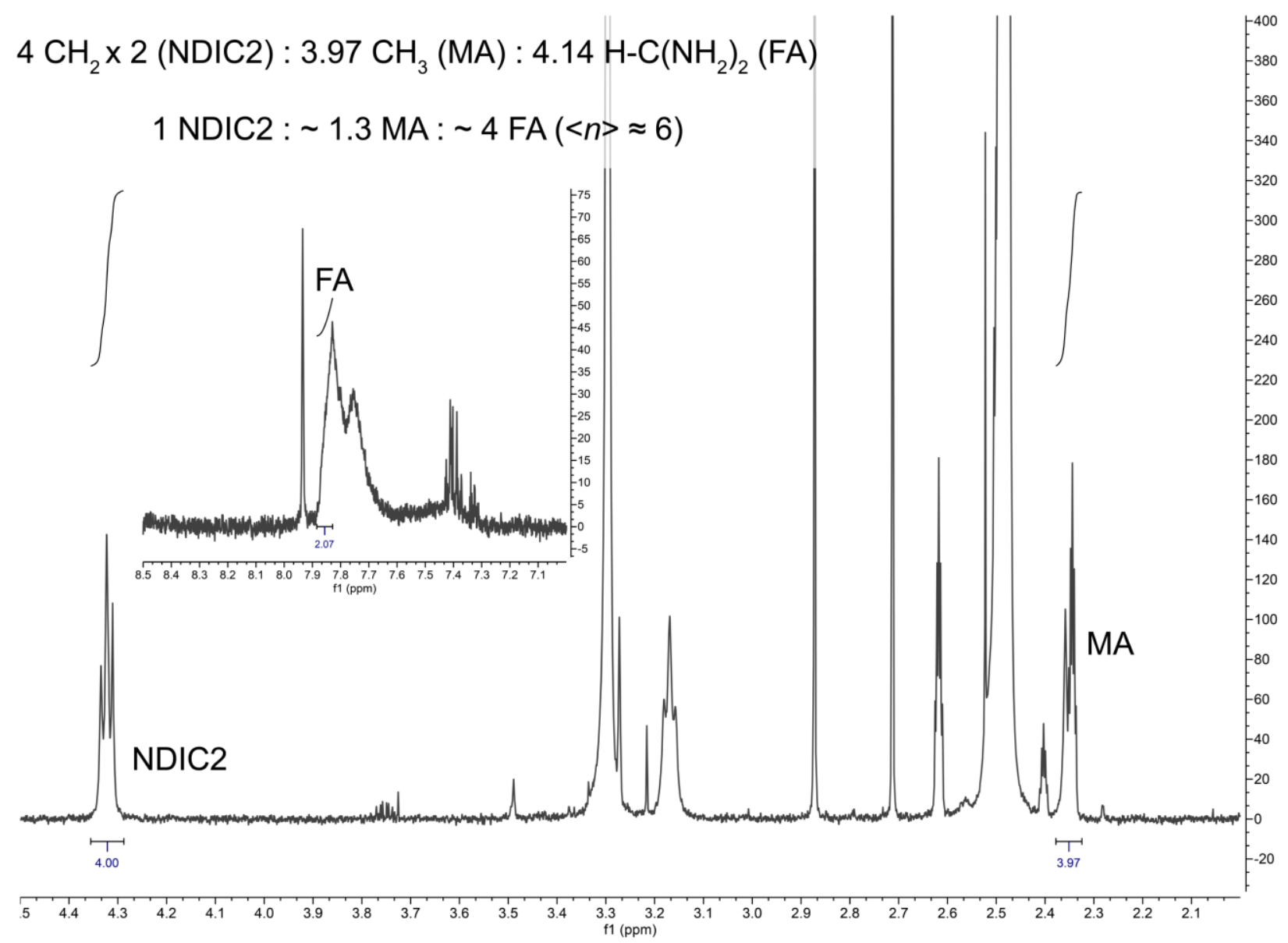

Figure S2. ${ }^{1} \mathrm{H}-\mathrm{NMR}$ spectrum of an $\langle n\rangle=5$ film with nominal formula (NDIC2)(FA $\left.{ }_{0.85} \mathrm{MA}_{0.15}\right)_{4} \mathrm{~Pb}_{5} \mathrm{I}_{16}$ dissolved in $d^{6}$-DMSO, and calculation of in-film $\langle n\rangle$ based on the cation ratio. Note the peak integration for FA at $\sim 7.8 \mathrm{ppm}$ was taken over half of the peak in order to avoid overlapping signal from other species, and then multiplied by 2 for the ratios. 


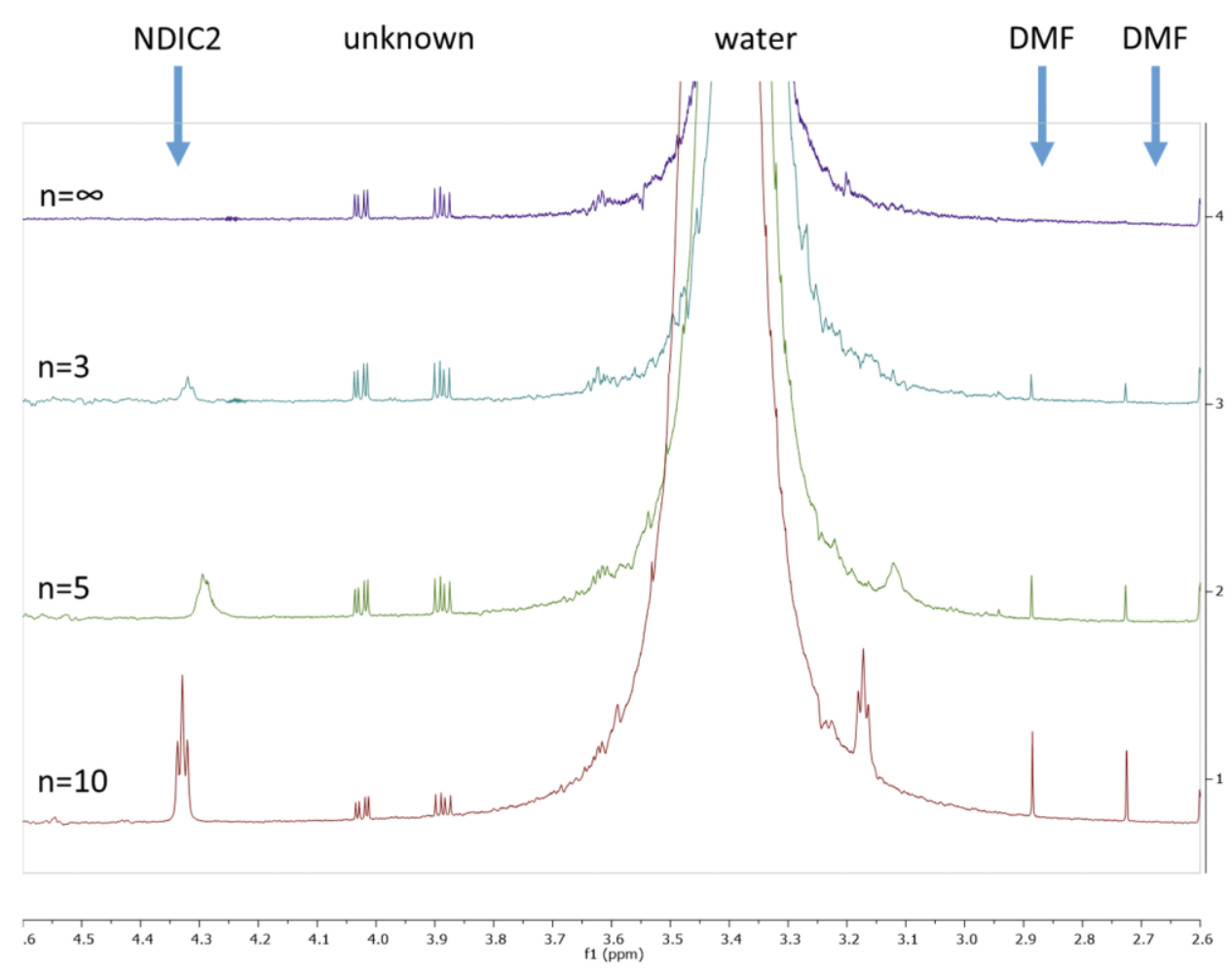

Figure S3. ${ }^{1} \mathrm{H}-\mathrm{NMR}$ spectra of various (NDIC2) ${ }_{2} \mathrm{MA}_{(n-1)} \mathrm{Pb}_{n} \mathrm{I}_{3 n+1}$ films redissolved into $d^{6}$-DMSO. Peaks arising from NDIC2, an unknown contaminant, water, and DMF are labelled. Films were annealed at $100^{\circ} \mathrm{C}$ for $15 \mathrm{~min}$. 
a

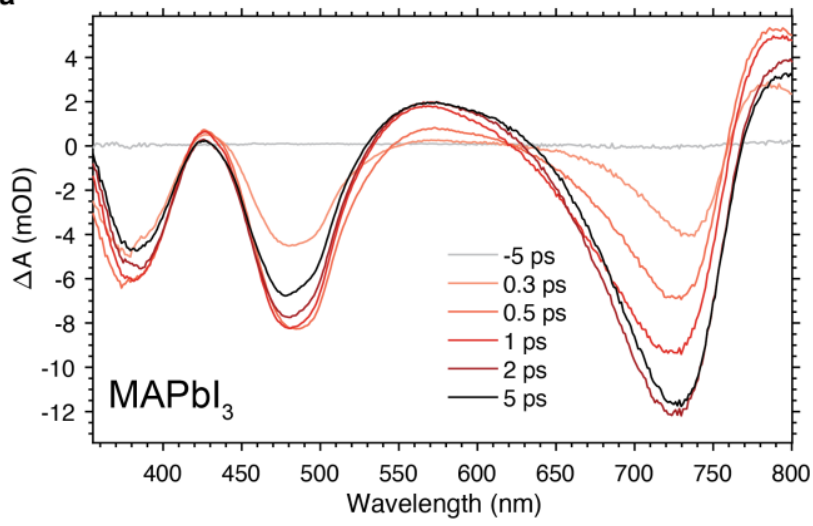

b

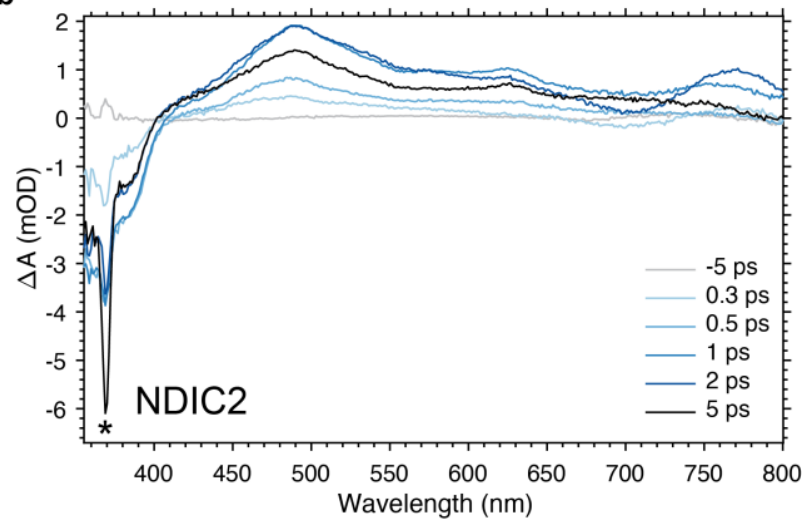

Figure S4. Transient absorption spectra following photoexcitation at $380 \mathrm{~nm}$ for thin films of (a) $\mathrm{MAPbI}_{3}$ and (b) NDIC2. Both films were prepared by dissolving precursors (or neat NDIC2) in DMF and spin-coating onto oxygen plasma treated glass slides. The asterisk indicates residual pump scatter that was not removed by background subtraction due to noise in the probe spectrum and sample translation during measurement. It is clear that the bleach peak of NDIC2 overlaps significantly with the second higher energy bleach peak of $\mathrm{MAPbI}_{3}$. 


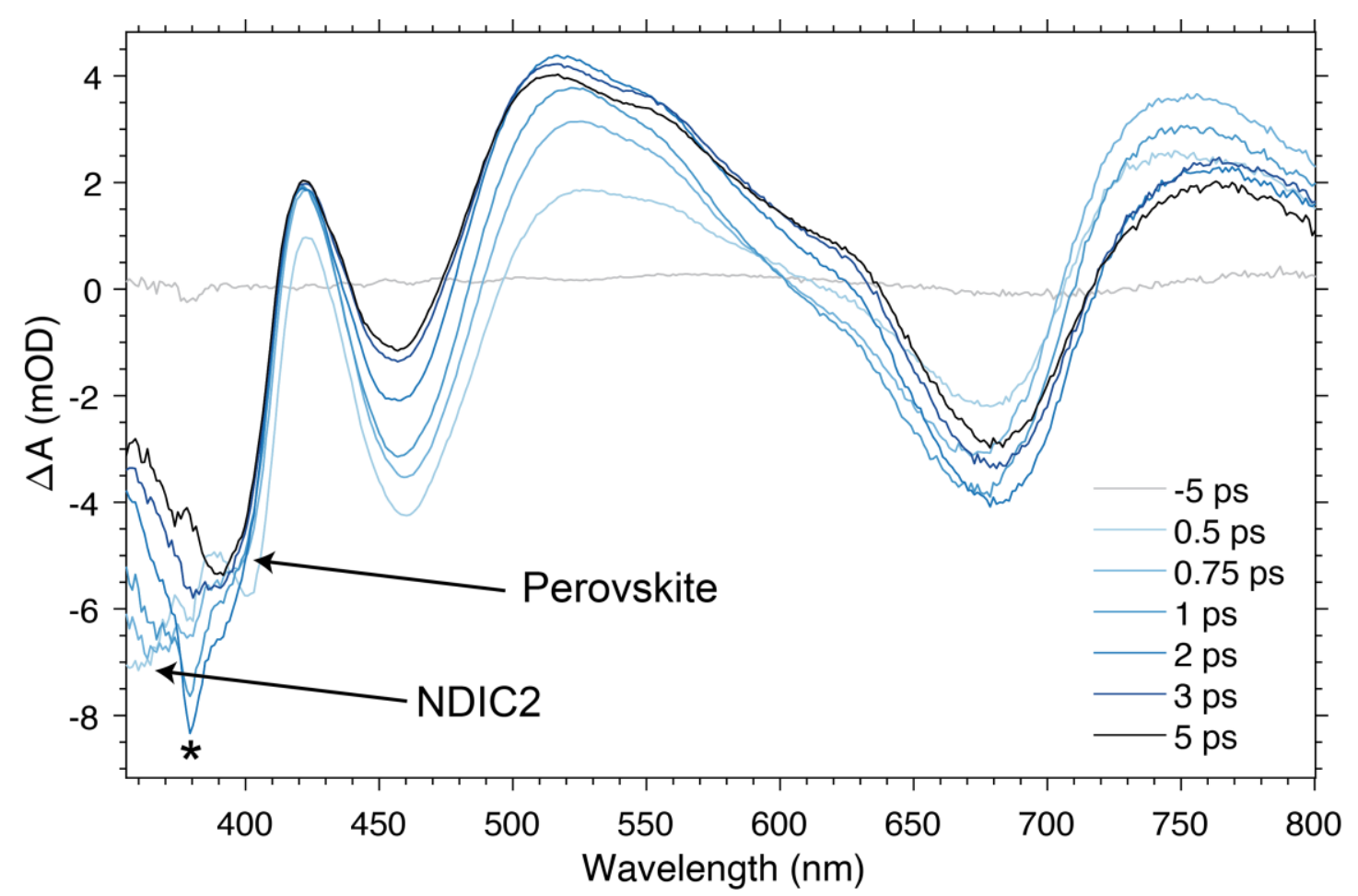

Figure S5. Transient absorption spectra for a thin film of (NDIC2) $\mathrm{MA}_{4} \mathrm{~Pb}_{5} \mathrm{I}_{16}$ (i.e. $\langle n\rangle=5$, FAfree) photoexcited at $380 \mathrm{~nm}$. Arrows indicate features arising from the NDIC2 molecule and from a higher energy bleach peak of quasi-3D perovskite. The asterisk indicates residual amplitude from pump scattering that was not fully removed at all time steps by background subtraction due to instability of the white light probe spectrum and translation of the substrate during measurements. While the bandedge bleach peak of the quasi-3D perovskite is blueshifted ( $675 \mathrm{~nm}$ instead of $\sim 740 \mathrm{~nm}$ ), this UV bleach peak appears to remain at the same energy as $\mathrm{MAPbI}_{3}$ (compared with Figure S4). A decay of the higher energy side of the peak (NDIC2 bleach) occurs simultaneously with a rise in the lower energy side of the peak (perovskite bleach), which is potentially indicative of population transfer; but probe spectrum noise and residual pump scattering partially obscure this region, and prevent a more definitive assignment of dynamics. 


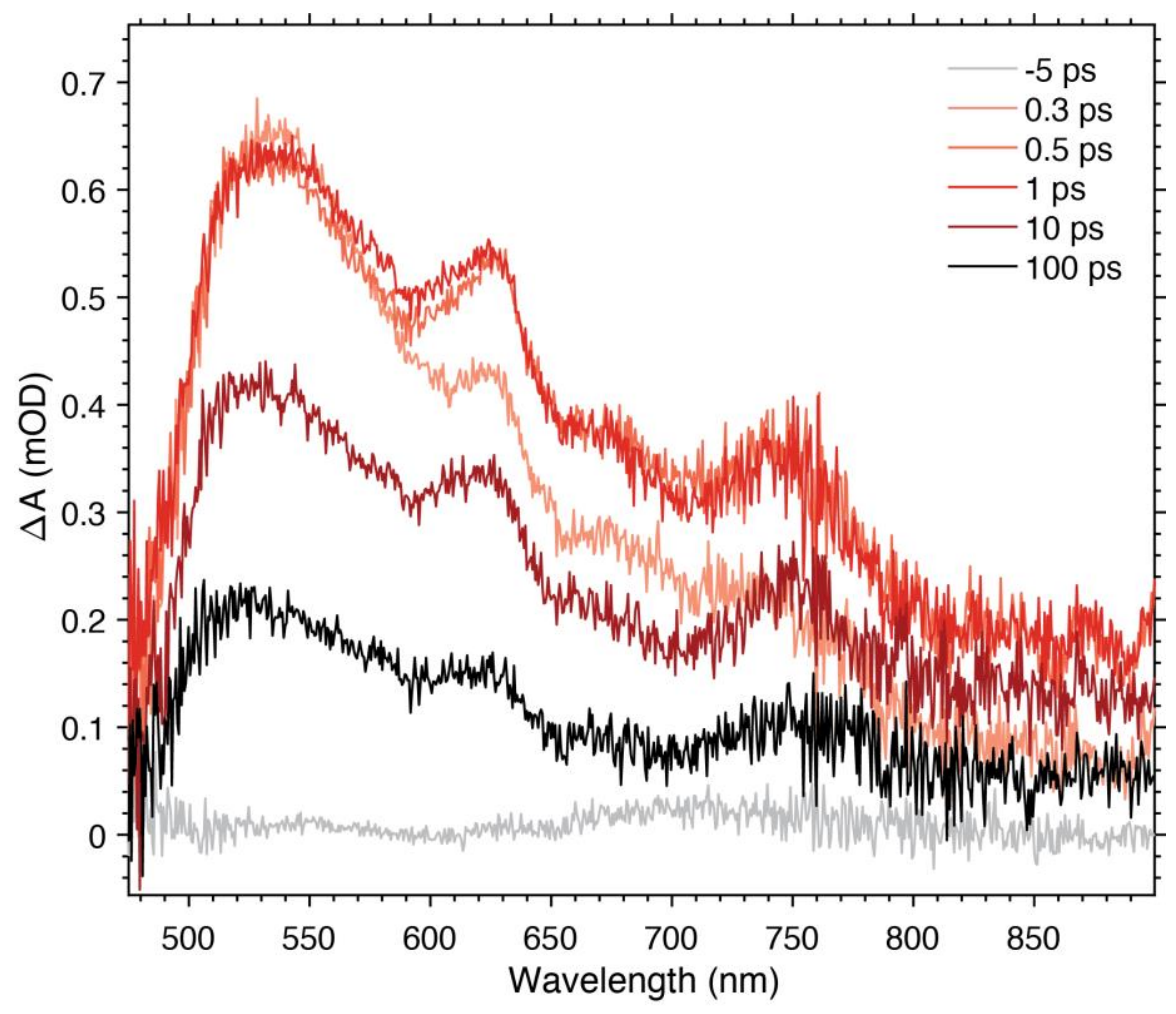

Figure S6. Transient absorption spectra following photoexcitation at $380 \mathrm{~nm}$ for a thin film NDIC2, prepared by dissolving NDIC2 in DMF and spin-coating onto an oxygen plasma treated glass slide. 

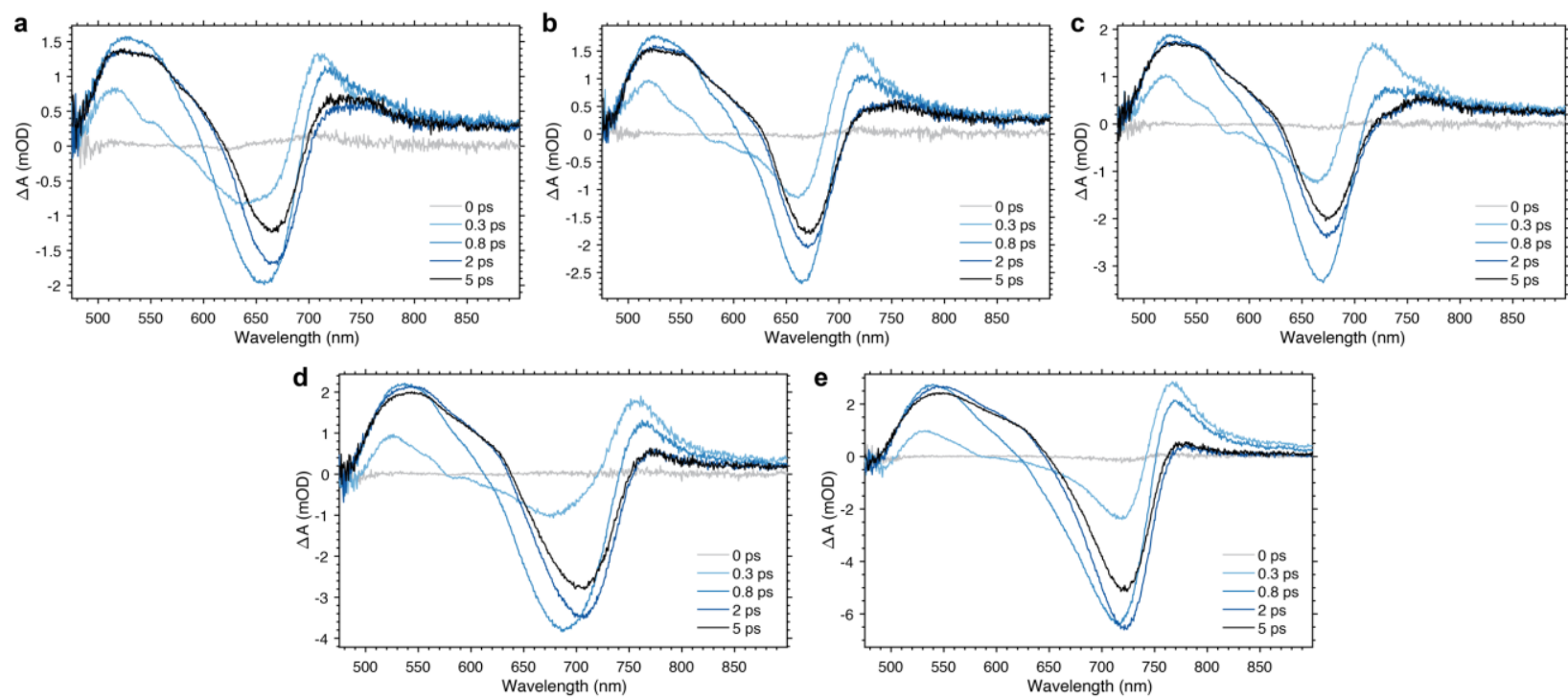

Figure S7. Transient absorption spectra for (NDIC2) $\mathrm{MA}_{(n-1)} \mathrm{Pb}_{n} \mathrm{I}_{3 n+1}$ films with $\langle n\rangle=4-8$. 

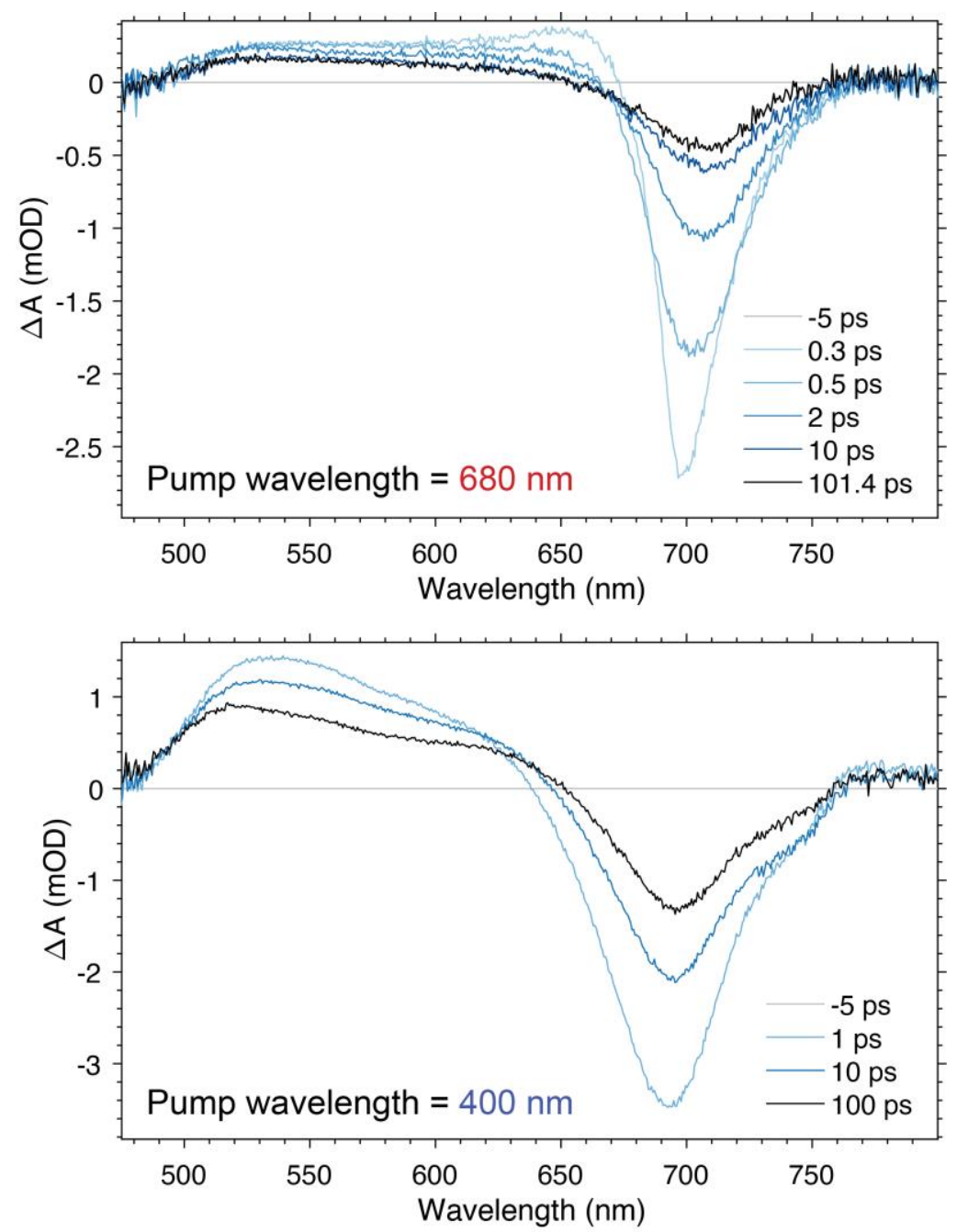

Figure S8. Transient absorption spectra for an (NDIC2) ${ }_{2} \mathrm{MA}_{4} \mathrm{~Pb}_{5} \mathrm{I}_{16}$ film following photoexcitation at $680 \mathrm{~nm}$ (top) and $400 \mathrm{~nm}$ (bottom). 

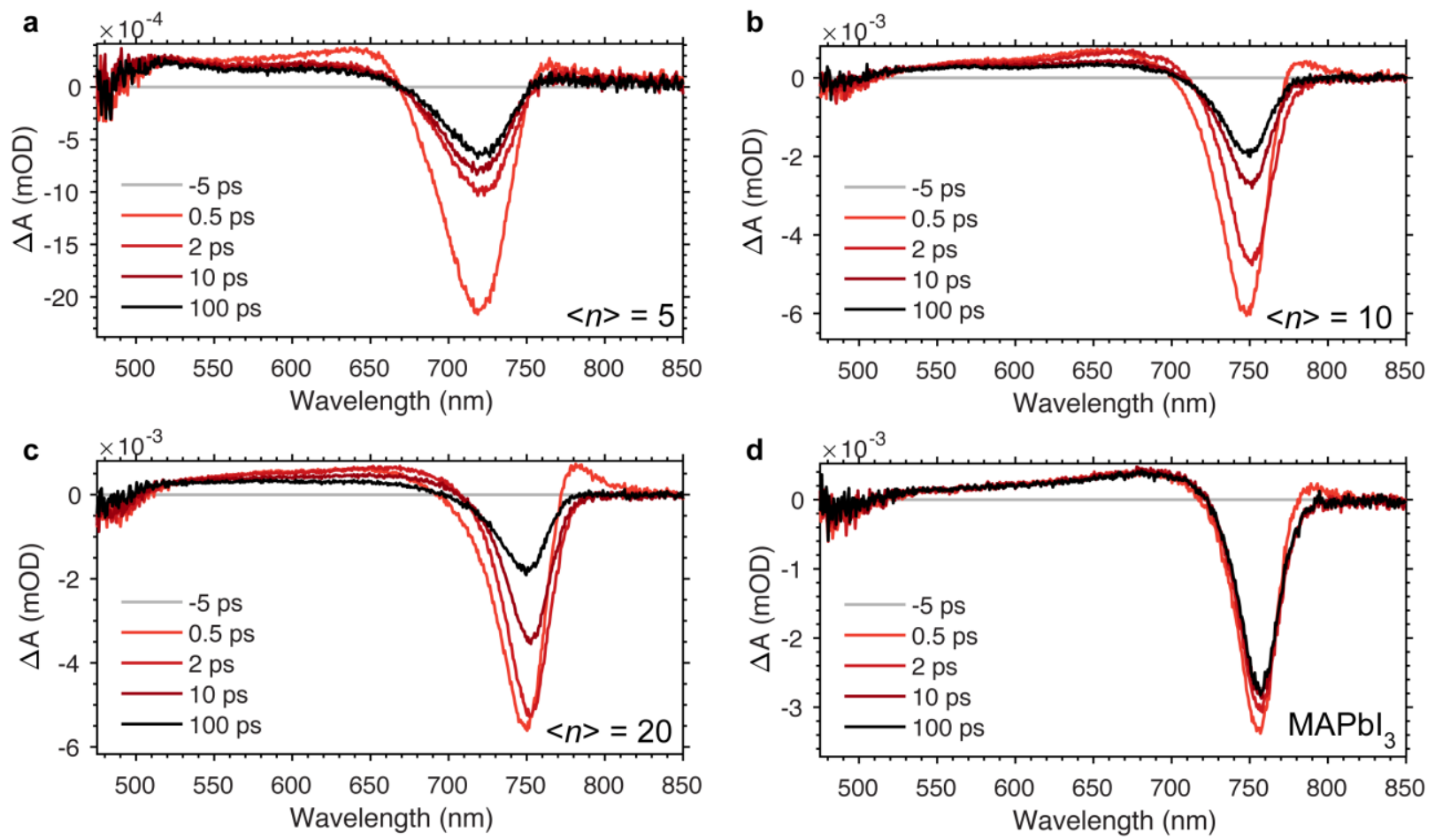

Figure S9. Transient absorption spectra at various delay times for (a) $\langle n\rangle=5$, (b) $\langle n\rangle=10$, (c) $\langle n\rangle$ $=20$, and (d) $\mathrm{MAPbI}_{3}$. All experiments were performed with a photoexcitation wavelength of 680 nm. 

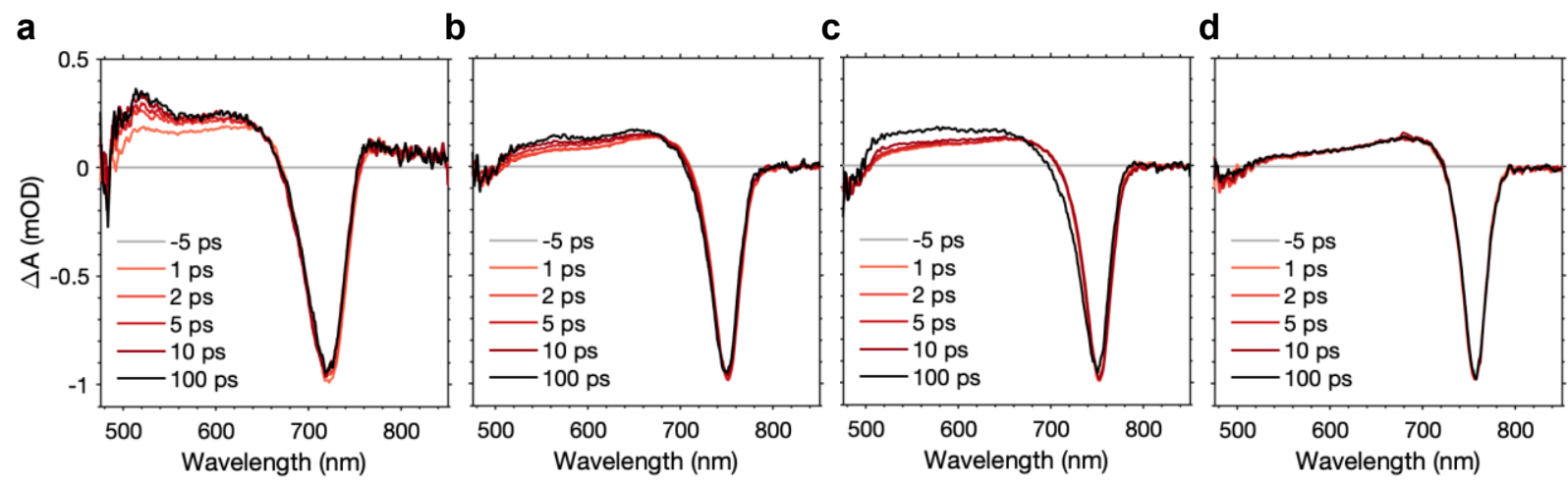

Figure S10. Transient absorption spectra for the (a) $\langle n\rangle=5$, (b) $\langle n\rangle=10$, (c) $\langle n\rangle=20$ and (d) $\mathrm{MAPbI}_{3}$ films, all normalized to -1 at their most negative value (bottom of the PQW bleach peak) for each time trace. The increasing amplitude between 500 and $650 \mathrm{~nm}$ is potentially indicative of electron transfer from the photoexcited perovskite to the NDIC2 ligands, as these spectral features and dynamics are completely absent for the $\mathrm{MAPbI}_{3}$ reference. Notably, the rate of the increasing amplitude over time is correlated with the rate of decay of the PQW bleach peak, i.e. it grows in fastest for $\langle n\rangle=5$, slowest for $\langle n\rangle=20$, further supporting assigning these dynamics to electron transfer from the PQW. 

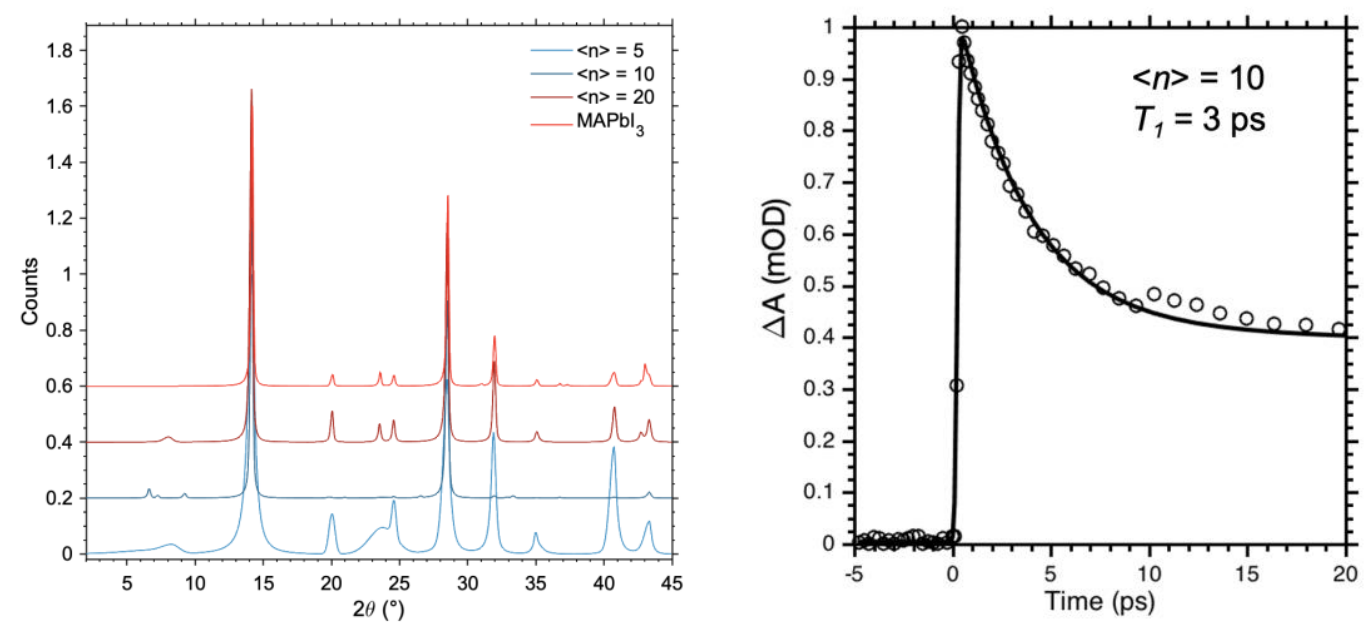

Figure S11. (left) XRD patterns for $\langle n\rangle=5,10,20$, and $\mathrm{MAPbI}_{3}$ films annealed at $70^{\circ} \mathrm{C}$ for 10 minutes rather than $100^{\circ} \mathrm{C}$ for 15 minutes. The peak at $8^{\circ}$ that we attribute to $1 \mathrm{D}$-like structures is either diminished $(\langle n\rangle=5$ and 20) or completely absent $(\langle n\rangle=10)$. TA experiments on these films yielded nearly identical dynamics. (right) Kinetic trace from the bandedge bleach peak of the $\langle n\rangle$ $=10$ film without any diffraction at $8^{\circ}$, showing a decay with a time constant of $\sim 3$ ps. The dynamics of the $\langle n\rangle=10$ film in the main text had a time constant of $\sim 3.3$ ps. 


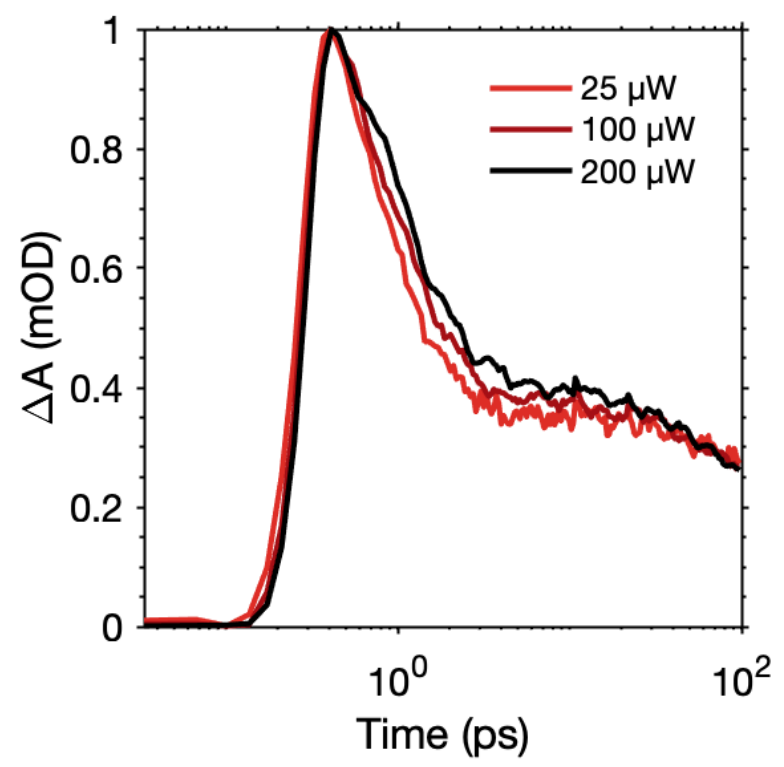

Figure S12. Kinetic traces taken from the bandedge exciton bleach in $\langle n\rangle=5$ films photoexcited at $680 \mathrm{~nm}$ with excitation powers of 25,100 and $200 \mu \mathrm{W}$. There are only minor differences in the dynamics for various excitation fluences, and the ultrafast decay component is still prominent for all cases. Unless otherwise stated, all transient absorption experiments using $680 \mathrm{~nm}$ photoexcitation were performed with a power of $100 \mu \mathrm{W}$, corresponding to an excitation fluence of $\sim 60 \mu \mathrm{Jcm}^{-2}$. 